\title{
Developing a Collaborative, Multi-Disciplinary Approach to the Supportive Care of Patients Undergoing External Beam Radiotherapy to the Perineum
}

\section{Introduction}

- External beam radiotherapy (EBRT) to the perineum is a common treatment for vulval and anal cancers.

- It is associated with a significant burden of early treatment-associated symptoms including skin toxicity, diarrhoea and pain.

- Symptoms often escalate throughout treatment and peak weeks after cessation of therapy.

- Quality of life can be affected and may result in premature cessation of therapy resulting in sub-optimal treatment.

-Where supportive care (SC) of treatment-related sideeffects is delayed or reactive, symptom control efforts may lag behind the escalating symptoms.
Challenges:

- Limited evidence-base

- No mechanism for co-ordinated SC throughout the patient journey across sites.

- Supportive and Palliative Care Team (SPCT) have insufficient capacity to provide supportive care for all such patients.

- Oncology helpline staff and Cancer Specialist Nurses have reported lower levels of confidence in managing these symptoms.

- Significant symptom burden with higher rates of unplanned admissions with uncontrolled symptoms.
The solution...

Service improvement project

Plan: Stakeholder focus-groups and ongoing feedback were used to develop a guideline for early, proactive, SC for all patients undergoing perineal radiotherapy.

Aim: All healthcare professionals along the patient journey to feel confident and equipped to provide consistent, timely, effective SC.

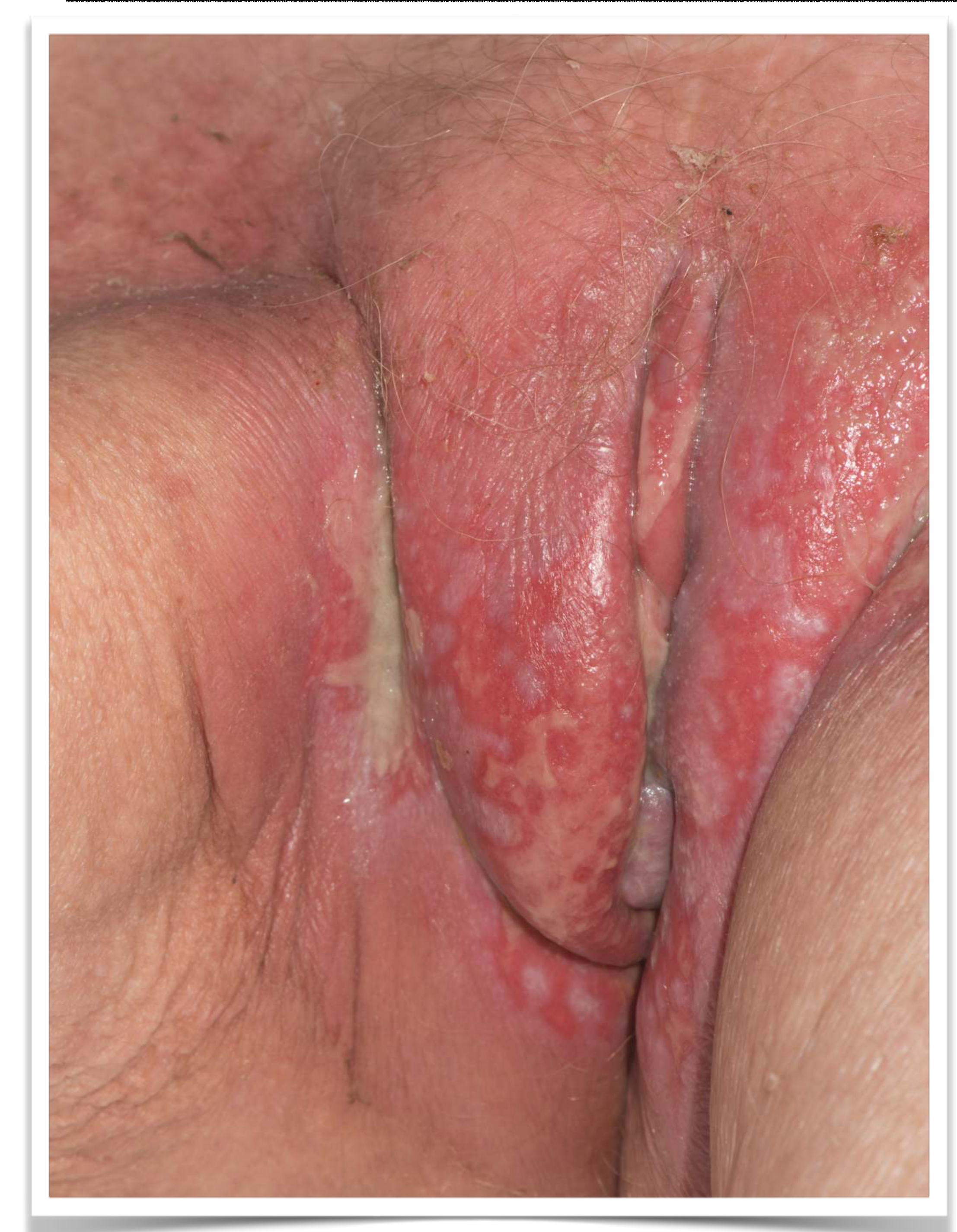

Severe radiotherapy skin toxicity with extensive moist desquamation

$\left\{\begin{array}{c}\text { "after I see them in clinic, they drop off } \\ \text { my radar for a few months... If they } \\ \text { have pain when I see them, I assume } \\ \text { their radiographers will manage it" } \\ \text { Gynaecology consultant }\end{array}\right.$

"We get calls about it occasionally but...don't really know what to expect or or Gynaecology oncology specialist

\section{t can be quite soul-sapping. You} and know they have weeks ahead yet...things are only going to get worse. You find yourself thinking, what more can I do other than help the patient keep treading water? Radiographer

\section{"It's one thing to know we should be} prescribing morphine but another to actually do it...you think "what happens they get worse because of the morphine?' Seeing it written down and knowing exactly what is expected of us... its given me much more confidence" Review radiographer

\section{Guideline Summary:}

Patient undergoing EBRT to the perineum

Anticipate significant pain during and after treatment

Pre-existing perineal pain or risk factors (eg fungating wound, higher dose EBRT) prescribe effective analgesia before patient begins EBRT

Communicate with GP to inform of upcoming treatment.

Ask for urgent referral to community occupational therapy for provision of pressurerelieving cushion. Patient encouraged to bring cushion to all future outpatient and therapy appointments.

\section{Prescription of strong opioid.}

All MDT prescribers should feel confident to prescribe symptom-control medications along the whole patient journey to ensure pro-active approach to SC. Titration and monitoring of analgesia throughout EBRT by review radiographers.

If inadequate symptom control at any stage: refer early to SPCT and inform clinical oncologist

\section{Prominent Incident Pain (eg on defecation or micturition):}

Pre-emptive topical lignocaine gel (eg Instillagel ${ }^{\circledR}$ ) (Monitor for signs of contact dermatitis)

Prominent superficial pain with desquamation (where oral opioids ineffective, nottolerated or contra-indicated): Regular topical morphine

\section{In cases of refractory symptoms, consider:}

- Secondary infection eg Candida spp. (particularly where sudden change in pain or exudate)

- Review skin care measures

- Reduction in boost/bolus to superficial tissues

- Additional domiciliary nursing/personal care

- Review medication concordance

- Admission for inpatient SC

- For EBRT with palliative-intent, consider stopping treatment

$$
\text { Be prepared to escalate analgesia up to two weeks after end of EBRT. }
$$

Personalised follow-up usually one to two weeks after the end of therapy: review ongoing symptom needs and de-escalate SC once symptoms improve.

Clear communication to patient and GP including treatment summary. Holistic

\section{needs assessment and ongoing monitoring for late effects}

"It's great to know what other ools we can use to manage [these symptoms] Review radiographer

"outside of a tertiary centre, these are quite rare cancers. We can go through a big part of our training without learning how to manage them Gynaecology registrar

"[the doctors] often don't look downbelow... they leave all that to us. I think it means that when it comes to [prescribing symptom-relief] they don't realise how bad it can be...they think codeine will be it can be...they think codeine will be enough. It's like 'out of sight, out of mind'”
Radiotherapy dept. nurse

"Its a really good idea. There is just too much need in radiotherapy for us to be everywher at once. this allows the other teams to be more proactive and manage the more raightforward cases themselves it allows us to focus our efforts on the more complex cases..."

Palliative care nurse specialist

\section{Next Steps:}

Audit of outcomes for all patients undergoing EBRT for vulval cancer, both before and after the guideline was implemented. Additional PDSA cycles depending on the results. We have developed a significant base of experience in using topical morphine in these patients - a case series is planned.

Development of training resources for new oncology doctors including photographs of typical examples of skin reactions along with training on management. 